STRUCTURAL BIOLOGY

ISSN 2059-7983

Keywords: data delivery; visualization; macromolecules; corrigendum

\section{High-performance macromolecular data delivery and visualization for the web. Corrigendum}

\author{
David Sehnal, ${ }^{\mathrm{a}, \mathrm{b}, \mathrm{c} *}$ Radka Svobodová, ${ }^{\mathrm{a}, \mathrm{b} *}$ Karel Berka, ${ }^{\mathrm{d}}$ Alexander S. Rose, \\ Stephen K. Burley, ${ }^{\mathrm{f} g, \mathrm{~h}}$ Sameer Velankar ${ }^{\mathrm{c}}$ and Jaroslav Koča ${ }^{\mathrm{a}, \mathrm{b}}$
}

${ }^{a}$ CEITEC - Central European Institute of Technology, Masaryk University, Kamenice 753/5, 62500 Brno, Czech Republic, ${ }^{\mathbf{b}}$ National Centre for Biomolecular Research, Faculty of Science, Masaryk University, Kamenice 753/5, 62500 Brno, Czech Republic, 'Protein Data Bank in Europe (PDBe), European Molecular Biology Laboratory, European Bioinformatics Institute (EMBL-EBI), Wellcome Genome Campus, Hinxton CB10 1SD, United Kingdom, ${ }^{\mathbf{d}}$ Regional Centre of Advanced Technologies and Materials, Department of Physical Chemistry, Faculty of Science, Palacký University Olomouc,

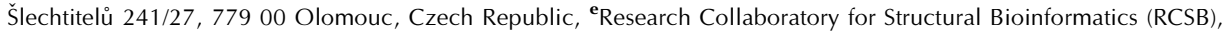
San Diego Supercomputer Center, University of California San Diego, 9500 Gilman Drive, La Jolla, San Diego, CA 92093-0743, USA, 'RCSB Protein Data Bank, Institute for Quantitative Biomedicine and Department of Chemistry and Chemical Biology, Rutgers, The State University of New Jersey, 174 Frelinghuysen Road, Piscataway, NJ 08854-8076, USA, ${ }^{\mathrm{g}}$ Cancer Institute of New Jersey, Rutgers, The State University of New Jersey, 195 Little Albany Street, New Brunswick, NJ 08903-2681, USA, and ${ }^{\mathbf{h}}$ RCSB Protein Data Bank, San Diego Supercomputer Center and Skaggs School of Pharmacy and Pharmaceutical Sciences, University of California San Diego, 9500 Gilman Drive, La Jolla, CA 920930654, USA. *Correspondence e-mail: david.sehnal@mail.muni.cz, radka.svobodova@ceitec.muni.cz

Two citations in the article by Sehnal et al. [(2020), Acta Cryst. D76, 1167-1173] are corrected.

In the Introduction section of the article by Sehnal et al. (2020) two incorrrect citations were given for PDB-Dev. The correct citations are Vallat et al. (2018) and Burley et al. (2017).

\section{References}

Burley, S. K., Kurisu, G., Markley, J. L., Nakamura, H., Velankar, S., Berman, H. M., Sali, S., Schwede, T. \& Trewhella, J. (2017). Structure, 25, 1317-1318.

Sehnal, D., Svobodová, R., Berka, K., Rose, A. S., Burley, S. K., Velankar, S. \& Koča, J. (2020). Acta Cryst. D76, 1167-1173.

Vallat, B., Webb, B., Westbrook, J. D., Sali, A. \& Berman, H. M. (2018). Structure, 26, 894-904.

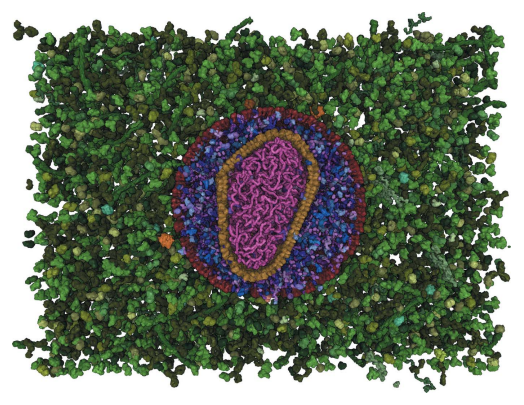

(c) (1) OPEN () ACCESS 